\title{
Computed distributions of rotovibrational transitions in $\mathrm{LiH}\left(\mathrm{X}^{1} \Sigma^{+}\right)$ and $\mathrm{LiH}^{+}\left(\mathrm{X}^{2} \Sigma^{+}\right)$
}

\author{
F.A. Gianturco ${ }^{1}$, P. Gori Giorgi ${ }^{1}$, H. Berriche ${ }^{2}$ and F.X. Gadea ${ }^{2}$ \\ 1 Department of Chemistry, The University of Rome, Cittá Universitaria, 00185 Rome, Italy \\ 2 Laboratoire de Physique Quantique, Université Paul Sabatier, 118 Rue de Narbonne, 31062 Toulouse Cedex, France
}

Received October 6; accepted November 21, 1995

\begin{abstract}
Accurate potential energy curves for the ground electronic states of $\mathrm{LiH}$ and $\mathrm{LiH}^{+}$are employed to generate vibrational and rotational levels over a broad range of $J$ values for both systems. The corresponding dipole functions are computed and used to obtain the frequency and intensity distributions of all relevant transitions between bound states for pure vibrational excitations, pure rotational excitations and for dipole allowed rovibrational processes. This extensive set of absorption data for both molecules is employed to locate the most likely region in the infrared spectrum where characteristic lines could be observed. Such data should be useful in experimental attempts to search for $\mathrm{LiH}$ and $\mathrm{LiH}^{+}$lines at high redshifts. We found that transitions between ionic levels will be markedly less intense than those for the neutral system and that transitions between rotationally 'hot' levels will be markedly more intense than those between low- $J$ levels.
\end{abstract}

Key words: molecular data - cosmology: miscellaneous

\section{Introduction}

According to current models of nucleosynthesis in the Hot Big-Bang (Peebles 1971; Wagoner et al. 1967) the first stars were formed from a gas consisting of hydrogen, helium and traces of deuterium $\left({ }^{2} \mathrm{H}\right),{ }^{3} \mathrm{He},{ }^{7} \mathrm{Li}$. In an environment where heavy elements or dust grains were absent, the important processes of cloud collapse by cooling and consequent stars formation need to be described quite differently from today. It has been suggested, therefore, that trace amounts of $\mathrm{H}_{2}$, formed from the $\mathrm{H}^{-}$ion, could furnish sufficient cooling to trigger cloud collapse (Hutchins 1976) provided that the molecules can remain undissociated by UV radiation or by collisions with $\mathrm{H}$ atoms. In other words, the radiative cooling at temperatures less than $8000 \mathrm{~K}$ is more generally considered to be dependent on the small fraction of the molecular components in the gas, since it is the molecular cooling that provides the prime mechanism for primordial cloud collapse. At the onset of the collapse it is therefore the particular structure of the molecular relative abundances, evolved from the earlier recombination, that can constrain the cooling process and therefore the onset of the galactic and stellar evolution of our universe (Lepp \& Shull 1984).
One of the important questions within that context is the role played by the abundances of $\mathrm{LiH}$ and $\mathrm{LiH}^{+}$, since it was suggested (Lepp \& Shull 1984) in a preliminary study of such systems that a rather large fraction of the primordial lithium might be incorporated into $\mathrm{LiH}$. The effect of a significant presence of $\mathrm{LiH}$ in the early universe has, in fact, been explored in recent years (Maoli et al. 1994) and found to be important, given the large value of the dipole moment of this molecule which allows interaction with photons of cosmic background radiation. Such studies suggested experimental attempts at observing in the region of radio frequencies (De Bernardis et al. 1993). The general chemistry of the early universe has been recently reviewed (Dalgarno \& Lepp 1987) and will not be repeated here: we will rather concentrate on the study of the structural and spectroscopic properties of two possible molecular candidates which would be playing a role in the cooling mechanism mentioned above.

The formation of the molecular ion $\mathrm{LiH}^{+}$can occur by the reaction

$$
\mathrm{Li}^{+}+\mathrm{H} \rightarrow \mathrm{LiH}^{+}+h \nu
$$

or by the alternative radiative association process

$$
\mathrm{Li}+\mathrm{H}^{+} \rightarrow \mathrm{LiH}^{+}+h \nu
$$


Table 1. Equilibrium distance $\left(R_{\text {eq }}\right)$, well depth $\left(D_{\mathrm{e}}\right)$, Zero Point Energy (ZPE) and transition energy $(\Delta E)$ for the $\mathrm{X}^{1} \Sigma^{+}$and $\mathrm{A}^{1} \Sigma^{+}$states of the LiH molecule

\begin{tabular}{|c|c|c|c|c|}
\hline $\mathrm{X}^{\mathrm{I}} \Sigma^{+}$ & $\mathrm{R}_{\mathrm{cq}}\left(\mathrm{a}, \mathrm{u}_{\mathrm{f}}\right)$ & $D_{e}\left(c m^{-1}\right)$ & $\mathrm{ZPE}\left(\mathrm{cm}^{-1}\right)$ & \\
\hline & 3011 & 2014898 & 690.76 & present work \\
\hline & 3.007 & 20174 & . & a \\
\hline & 3.021 & 19972 & - & b \\
\hline & 3.050 & 19445 & - & $c$ \\
\hline & 3.049 & 18794 & - & $d$ \\
\hline & 3.015 & 18228 & - & $e$ \\
\hline & 3.009 & 19559 & . & $\mathrm{f}$ \\
\hline & 3,081 & 20100 & - & $\mathrm{g}$ \\
\hline & 3.066 & 18367.48 & . & $\mathrm{h}$ \\
\hline & 3.015 & 20287.7 & 697.90 & Expt. $(i, j, k)$ \\
\hline & & & $\Delta \mathrm{E}\left(\mathrm{cm}^{-1}\right)$ & \\
\hline $\mathrm{A}^{15+}$ & 4.857 & 8677.78 & 26377 & present work \\
\hline & 4.847 & 8689.7 & 26389 & a \\
\hline & 4.783 & 9042 & 26212 & $b$ \\
\hline & 5.120 & 8451 & 25842 & $\mathrm{c}$ \\
\hline & 4.679 & 8212 & 25145 & d \\
\hline & 4.901 & 8630 & - & $e$ \\
\hline & 4.910 & 8444 & - & f \\
\hline & 4.800 & 9040 & - & $g$ \\
\hline & 4.042 & 11151.5 & - & $\mathrm{h}$ \\
\hline & 4.907 & 8682 & 26509 & $\operatorname{Expt}(i, j, l)$ \\
\hline
\end{tabular}

a) Boutalib \& Gadea 1992; b) Partridge \& Langhoff 1981; c) Docken \& Iinze 1972;

d) Sasagane et al. 1990: el Wang et al. 1989; f) Ben-Shlomo et al. 1988; g) Mendez et al. 1990; h) Mo et al. 1993; i) Way et al. 1973; j) Huber et al. 1978; k) Chan et al. 1986; 1) Stwalley et al. 1993.

Table 2. Bound vibrational levels $(J=0)$ for the $\mathrm{X}^{1} \Sigma^{+}$state of LiH computed with the present PEC

\begin{tabular}{|c|c|c|c|c|c|}
\hline $\mathrm{v}$ & $\begin{array}{r}\text { Level }\left(\mathrm{cm}^{-1}\right) \\
\text { Position }\end{array}$ & $\begin{array}{r}\mathrm{E}(\mathrm{v}+\mathrm{l})-\mathrm{E}(\mathrm{v}) \\
\left.(\mathrm{ctr})^{-1}\right) \\
\text { Present work }\end{array}$ & $\begin{array}{r}\text { Eiv+l)-E(v) } \\
\left(\mathrm{cm}^{-1}\right) \\
\text { Partridge } 1981\end{array}$ & $\begin{array}{r}\mathrm{E}(\mathrm{v}+1)-\mathrm{E}(\mathrm{v}) \\
(\mathrm{cm}-1) \\
\text { Exp; Way } 1973\end{array}$ & $\begin{array}{r}\mathrm{E}(\mathrm{v}+1)-\mathrm{E}(\mathrm{v}) \\
\left(\mathrm{cm}^{-1}\right) \\
\mathrm{Exp}_{7} \text { Chan } 1986\end{array}$ \\
\hline 0 & 19458.22 & 1324.88 & 1352.9 & 1359.76 & 1359.71 \\
\hline 1 & 18133.34 & 1320.94 & 1309.0 & 1314.85 & 1314.89 \\
\hline 2 & 16812.40 & 1258.16 & 1266.5 & 1270.87 & 1270.89 \\
\hline 3 & 15554.24 & 1216.15 & 1223.6 & 1227.82 & 1227.77 \\
\hline 4 & 14338.09 & 1174.56 & 1180.1 & 1185.40 & 1185.44 \\
\hline 5 & 13163.53 & 1133.46 & 1135.6 & 1143.83 & 1143.77 \\
\hline 6 & 12030.07 & 1092.96 & 1091.4 & 1102.63 & 1102.6 \\
\hline 7 & 10937.11 & 1052.89 & 1050.9 & 1061.74 & 1061.78 \\
\hline 8 & 9884.22 & 1013,00 & 1009.6 & 1021.16 & 1021.17 \\
\hline 9 & 8871.22 & 973.04 & 969.9 & 980.42 & 980.52 \\
\hline 10 & 7898.18 & 932.74 & 929.4 & 939.97 & 939.62 \\
\hline 11 & 6965.44 & 891.82 & 888.3 & 897.97 & 898.09 \\
\hline 12 & 6073.62 & 849.80 & 845.5 & & 855.48 \\
\hline 13 & 5223.82 & 806.08 & 800.0 & & 811.19 \\
\hline 14 & 4417.74 & 759.92 & 751.8 & & 764.44 \\
\hline 15 & 3657.82 & 710.41 & 700.0 & & 714.22 \\
\hline 16 & 2947.41 & 656.18 & 643.1 & & 659.27 \\
\hline 17 & 2291.23 & 595.51 & 578.5 & & 597.89 \\
\hline 18 & 169572 & 526.47 & 503.1 & & 527.91 \\
\hline 19 & 1169.25 & 446.35 & 415.1 & & 446.61 \\
\hline 20 & 722.90 & 352.34 & 312.0 & & 350.99 \\
\hline 21 & 370.56 & 242,10 & 189.5 & & 237.71 \\
\hline 22 & 128.46 & 116.24 & & & \\
\hline 23 & 12.22 & & & & \\
\hline
\end{tabular}

a) exp1. Irom Stwalley et al. $1993 ; 19589.8 \mathrm{~cm}^{-1}$. 

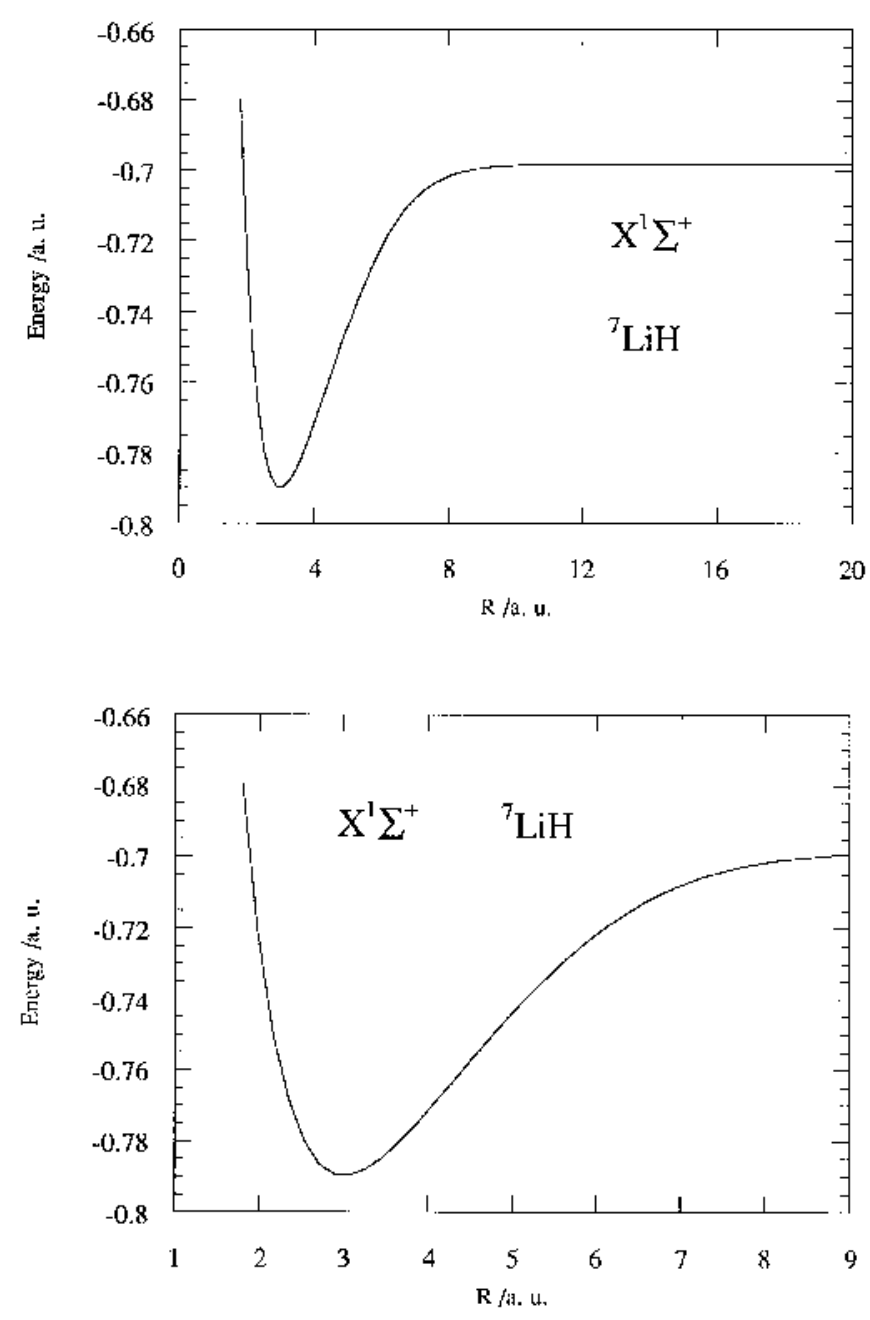

Fig. 1. Computed potential energy curve $\mathrm{X}^{1} \Sigma^{+}$electronic state of the LiH molecule. All quantities are in atomic units. The lower part shows the well region of the potential

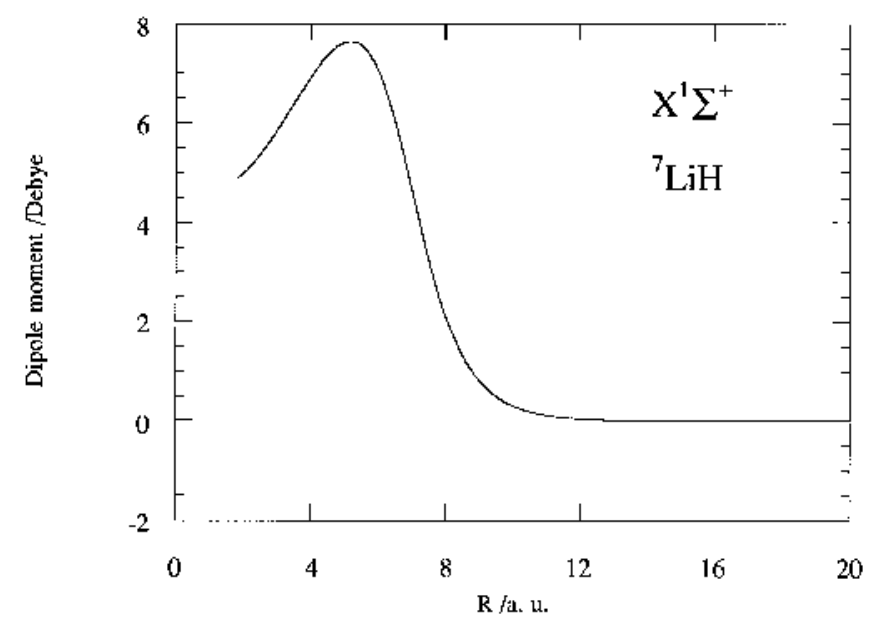

Fig. 2. Computed dipole moment for the $\mathrm{X}^{1} \Sigma^{+}$electronic state of $\mathrm{LiH}$ as a function of internuclear distance. The values are in Debye while the distance is given in atomic units 
Table 3. Computed and measured rotational transitions for the $\mathrm{X}^{1} \Sigma^{+}$state of $\mathrm{LiH}($ data in $\mathrm{MHz}$ )

\begin{tabular}{|c|c|c|c|c|c|}
\hline \multicolumn{6}{|c|}{ Transition } \\
\hline & $J-J^{\prime}$ & Present calc.s & Exp. (Bellini 1994) & Absolute Error & $\%$ Error \\
\hline$v=0$ & $0-1$ & 446450.9285 & 443952.93 & 2497.99 & 0.56 \\
\hline$v=0$ & $1-2$ & 892272.2927 & & & \\
\hline$v=0$ & $2-3$ & 1336804.549 & 1329415.425 & 7389.12 & 0.56 \\
\hline$v=0$ & $3-4$ & 1779448.114 & 1769711.222 & 9736.89 & 0.55 \\
\hline$v=0$ & $4-5$ & 2219573.421 & 2207584.583 & 11988.83 & 0.54 \\
\hline$v=0$ & $5-6$ & 2656580.887 & & & \\
\hline$v=0$ & $6-7$ & 3089810.968 & 3073719.689 & 16091.27 & 0.52 \\
\hline$v=0$ & $7-8$ & 3518754.017 & & & \\
\hline$v=1$ & $0-1$ & 434189.4169 & & & \\
\hline$v=1$ & $1-2$ & 867719.2904 & & & \\
\hline$v=1$ & $2-3$ & 1300020.015 & & & \\
\hline$v=1$ & $3-4$ & 1730432.047 & & & \\
\hline$v=1$ & $4-5$ & 2158385.781 & 2144133.66 & 14252.12 & 0.66 \\
\hline$v=1$ & $5-6$ & 2583221.673 & 2566428.32 & 16793.35 & 0.65 \\
\hline$v=1$ & $6-7$ & 3004400.097 & & & \\
\hline$v=1$ & $7-8$ & 3421291.489 & & & \\
\hline$v=2$ & $0-1$ & 421897.9261 & & & \\
\hline$v=2$ & $1-2$ & 843226.2466 & & & \\
\hline$v=2$ & $2-3$ & 1263265.46 & 1253771.89 & 9493.57 & 0.76 \\
\hline$v=2$ & $3-4$ & 1681505.918 & & & \\
\hline$v=2$ & $4-5$ & 2097288.078 & & & \\
\hline$v=2$ & $5-6$ & 2510042.334 & 2491759.62 & 18282.71 & 0.73 \\
\hline$v=2$ & $6-7$ & 2919169.101 & & & \\
\hline$v=2$ & $7-8$ & 3324128.754 & & & \\
\hline
\end{tabular}

Table 4. Computed and measured values of the dipole moment function for the $\mathrm{X}^{1} \Sigma^{+}$electronic state of LiH. Distances are in a.u. and $\mu$ is given in Debye. The polarity is that of $\mathrm{Li}^{+} \mathrm{H}^{-}$with respect to the center of mass of the molecule. Only some representative values are reported for comparison

\begin{tabular}{|c|c|c|c|}
\hline $\begin{array}{l}\text { Distance /an } \\
1.8\end{array}$ & $\begin{array}{l}\text { Present cale.s } \\
4.478\end{array}$ & $\begin{array}{l}\text { Patridge } 1981 \\
\text {. }\end{array}$ & $\begin{array}{l}\text { Dokken } 1972 \\
-\end{array}$ \\
\hline $\begin{array}{l}1.0 \\
2.0\end{array}$ & 4.974 & 4.952 & 5.004 \\
\hline 2.5 & 5.344 & 5.322 & 5.378 \\
\hline 3.0 & 5.832 & 5.811 & 5.872 \\
\hline 4.0 & 6.905 & 6.882 & 6.952 \\
\hline 5.0 & 7.616 & 7.565 & 7.685 \\
\hline 6.0 & 7,073 & 6.933 & 7.163 \\
\hline 7.0 & 4.678 & 4.466 & \\
\hline 8.5 & 1.323 & 1.226 & 1.310 \\
\hline 10.0 & 0.295 & 0.278 & 0.287 \\
\hline 12.0 & 0.0365 & 0.0478 & 0.0422 \\
\hline 15.0 & 0.00179 & 0.0112 & - \\
\hline 20.0 & 0.00268 & 0.000266 & - \\
\hline
\end{tabular}

With respect to the production of neutral lithium, $\mathrm{LiH}$ can then be formed by radiative association

$$
\mathrm{Li}+\mathrm{H} \rightarrow \mathrm{LiH}+h \nu
$$

or by associative detachment

$$
\mathrm{Li}+\mathrm{H}^{-} \rightarrow \mathrm{LiH}+\mathrm{e}^{-}
$$

The neutral molecule can also undergo charge transfer reactions

$$
\mathrm{LiH}+\mathrm{H}^{+} \rightarrow \mathrm{LiH}^{+}+\mathrm{H}
$$

(3) which produce further $\mathrm{LiH}^{+}$, plus various other reactions that will deplete its presence in the earlier clouds, as it will also occur to $\mathrm{LiH}^{+}$through an additional series of different mechanisms. All of them have been recently discussed (Stancil et al. 1995) and therefore the details will not be repeated here. Suffice it to say that the relative abundance 
Table 5. A comparison of computed and measured expectation values of the dipole moment $\langle\mu\rangle$ for all the bound vibrational levels for the $\mathrm{X}^{1} \Sigma^{+}$electronic state of ${ }^{7} \mathrm{LiH}$. All values in atomic units (all values for $J=0$ )

\begin{tabular}{|lllll|}
\hline$y$ & This work & Zernke 1980 & Expt. (a) & Expt (b) \\
0 & 2.3194 & 2.3380 & 2.314 & 2.314 \\
1 & 2.3612 & 2.3811 & 2.357 & \\
2 & 2.4026 & 2.4226 & 2.399 & \\
3 & 2.4436 & 2.4463 & & \\
4 & 2.4845 & 2.5104 & Docken 1972 & \\
5 & 2.5247 & 2.5518 & 2.559 & \\
6 & 2.5631 & 2.5928 & & \\
7 & 2.5987 & 2.6311 & & \\
8 & 2.6305 & 2.6651 & & \\
9 & 2.6573 & 2.6933 & & \\
10 & 2.6779 & 2.7145 & 2.711 & \\
11 & 2.6907 & 2.7269 & & \\
12 & 2.6935 & 2.7283 & & \\
13 & 2.6835 & 2.7156 & & \\
14 & 2.6570 & 2.6852 & & \\
15 & 2.6091 & 2.6320 & & \\
16 & 2.5333 & 2.5489 & & \\
17 & 2.4209 & 2.4269 & & \\
18 & 2.2608 & 2.2550 & & \\
19 & 2.0389 & 2.0183 & & \\
20 & 1.7387 & 1.6942 & & \\
21 & 1.3434 & 1.2645 & & \\
22 & 0.8411 & 0.7063 & & \\
23 & 0.2551 & 0.0448 & & \\
\hline
\end{tabular}

a) Rothstein 1969; b) Wiese et al. 1966
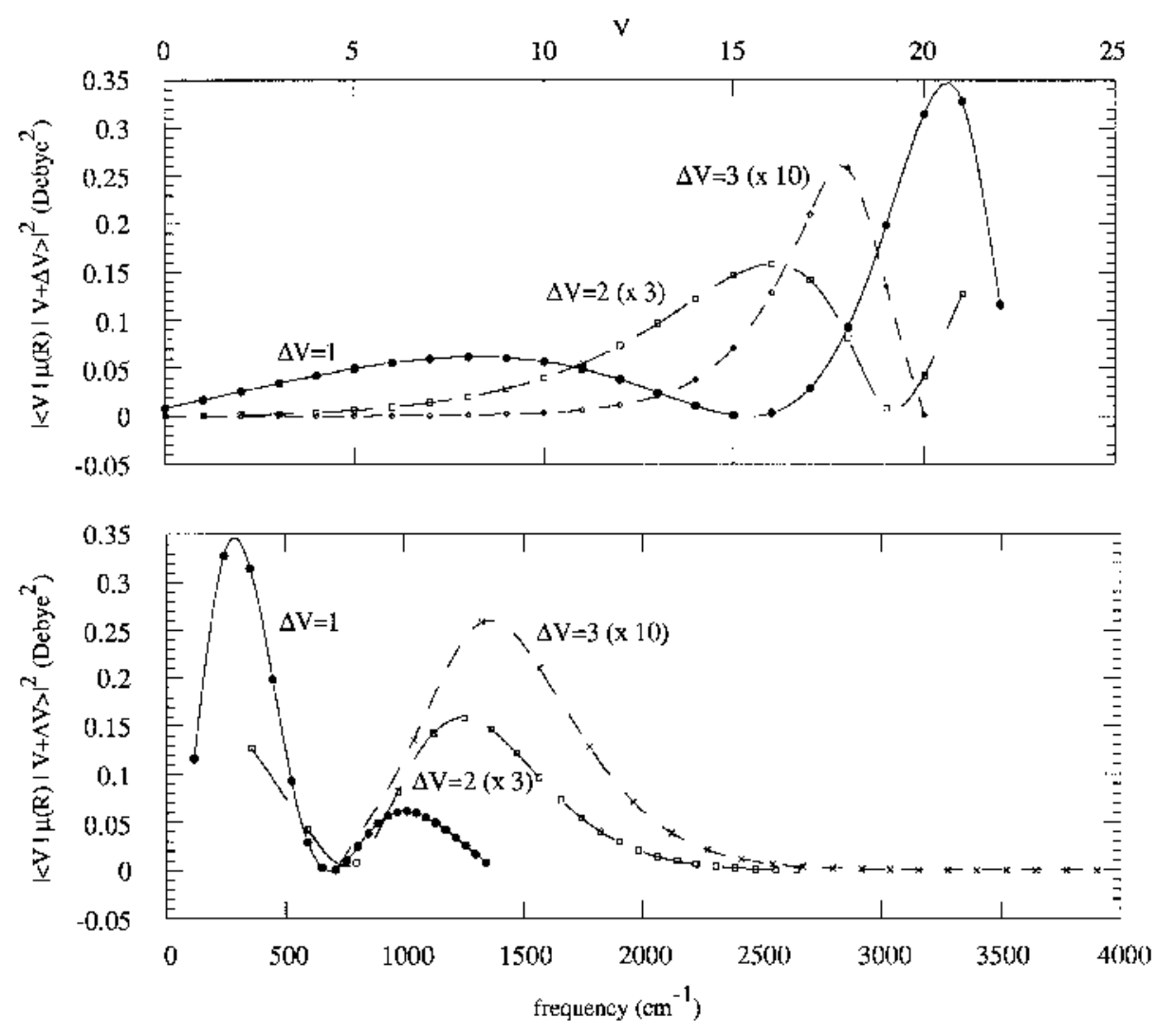

Fig. 3. Computed transition moments between bound vibrational levels $(J=0)$ of the $\mathrm{X}^{1} \Sigma^{+}$state of LiH. Top: transitions as functions of initial vibrational state. Bottom: transitions as a functions of frequency. The multiple-jump transitions are multiplied by different scale factors to make them more visible 


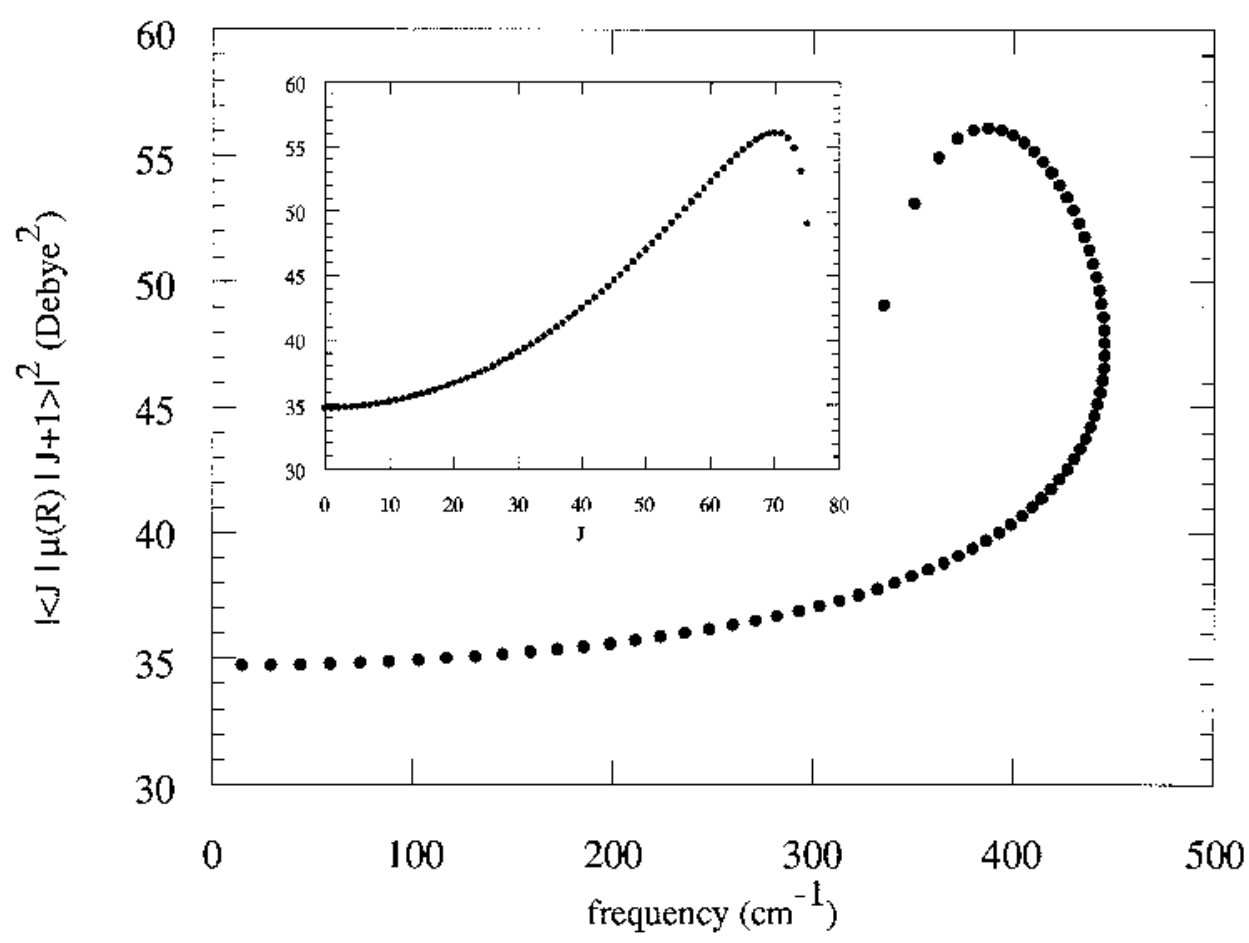

Fig. 4. Computed transition moments for pure rotational transitions within the ground vibrational level of the $\mathrm{X}^{1} \Sigma^{+}$electronic state of $\mathrm{LiH}$. The inset shows the intensity as a function of initial rotational state

of $\mathrm{LiH}^{+}$is predicted to be $10 \%$ of that for neutral $\mathrm{LiH}$ for small redshift values, while becoming much smaller as the redshift increases.

The detailed study of the reactions (1) to (4) requires a reliable knowledge of the potential energy surfaces associated with the electronic states which would be relevant to astrophysical observation and a quantitative knowledge of their bound rotovibrational states, of their transition moments and hence of the possible observational windows through which the presence of such species would be more likely to be detected. This is the object of the present work, in which we examine the distribution of transition probabilities and absorption coefficients for vibrational, rotational and rovibrational transitions between the bound states of the lowest electronic potentials of $\mathrm{LiH}$ and $\mathrm{LiH}^{+}$.

We will also discuss the present calculations in relation to avalaible experiments and to previous existing calculations for the same systems.

The following section will report the results obtained for the $\mathrm{LiH}$ molecule, while Sect. 3 will carry out the same analyisis for $\mathrm{LiH}^{+}$. The general conclusions and suggestions for experimental observations will be presented in Sect. 4. We will therefore focus our attention on the rotational-vibrational (RV) transitions in both systems and describe below the theoretical and computational methods we employed in order to provide a rather detailed study on such structures.

\section{The structure of RV lines in neutral $\mathrm{LiH}$}

\subsection{The potential energy curve (PEC)}

Several calculations have been carried out over the years on the ground and excited electronic states of the $\mathrm{LiH}$ molecule because of its simple electronic structure and of its interest in many different fields of research.

The earlier theoretical work concentrated mainly on the ground electronic state and on the dipole moment value at equilibrium geometries. For example, the GVB method was employed (Palke \& Goddard 1969) to discuss some of the bonding features of $\mathrm{LiH}$. Later on, a general Multiconfiguration Hartree-Fock (MCHF) procedure was used (Docken \& Hinze 1969; hereafter D \& H) to carefully investigate five valence states and to show for the first time that the $\mathrm{B}^{1} \Pi$ state is weakly bound. Further, extensive CI calculations were undertaken (Partridge \& Langhoff 1981; hereafter $\mathrm{P} \& \mathrm{~L}$ ) on the $\mathrm{X}^{1} \Sigma^{+}, \mathrm{A}^{1} \Sigma^{+}$, and $\mathrm{B}^{1} \Pi$ states using a $22 \sigma 12 \pi 7 \delta$ STO basis set, obtaining dissociation energies close to the experimental values, while also analysing dipole moments and transition moments between these states. More recently, several other calculations were carried out on the $\mathrm{X}^{1} \Sigma^{+}$and the $\mathrm{A}^{1} \Sigma^{+}$states (Sasagane et al. 1990; Wang et al. 1989; Ben-Shlomo et al. 1988; Mendez et al. 1990) and on various spectroscopic properties of these two electronic states. A rather extensive comparison with their results was presented by Boutalib \& Gadea (Boutalib \& Gadea 1992), who carried out the first adiabatic and 

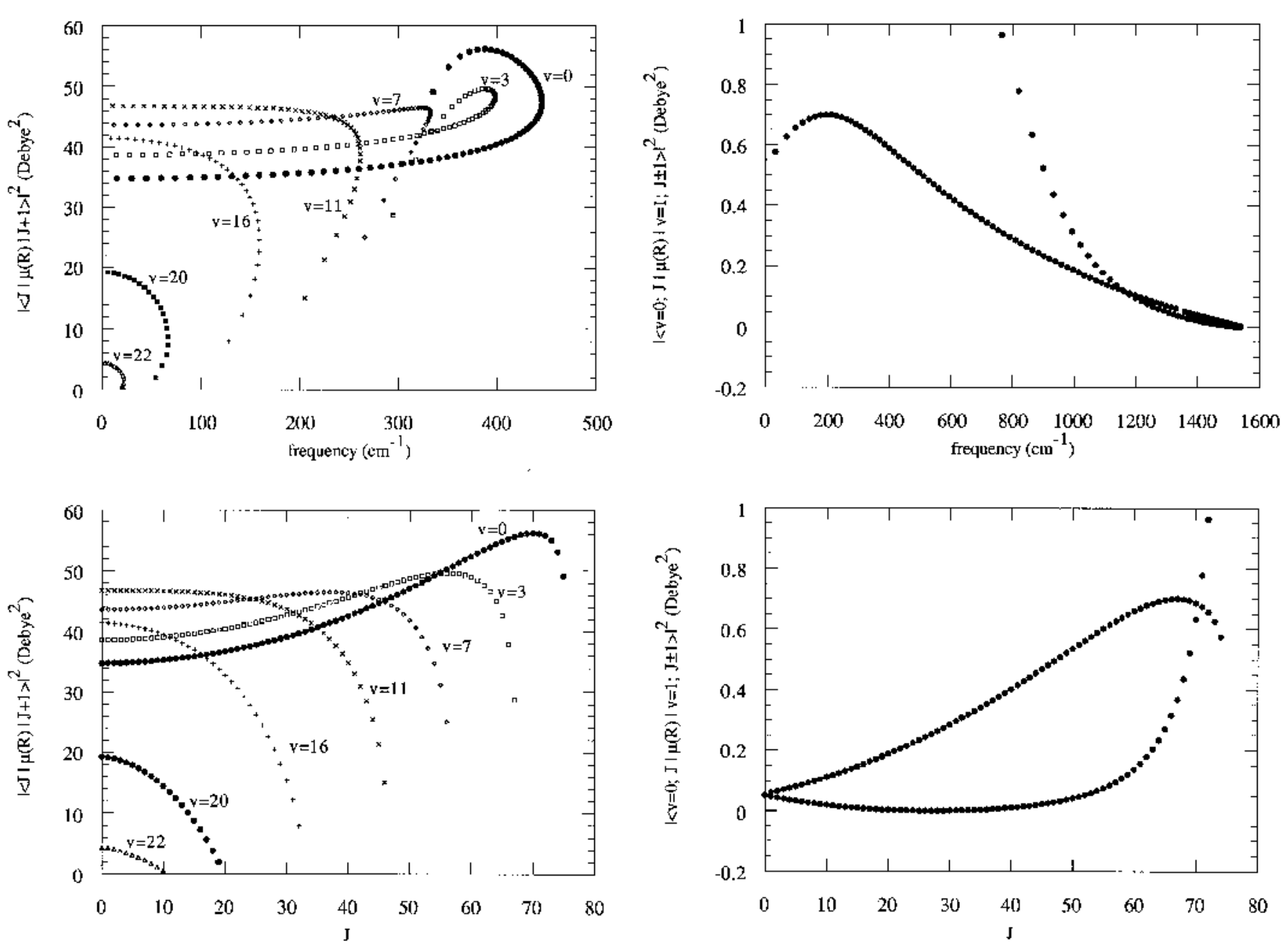

Fig. 5. Pure rotational transition moments as a functions of frequency (top) and of initial rotational state $J$ (bottom) and for various vibrational bound states of the $\mathrm{X}^{1} \Sigma^{+}$state of the $\mathrm{LiH}$ molecule

diabatic state analysis for nearly all the electronic states below the ionisation limit for the entire $\mathrm{LiH}$ potential energy curve. Their treatment involved a non-empirical pseudopotential for Li and a full configuration-interaction (CI) treatment of the valence electrons. Core-valence correlation effects were taken into account following a corepolarisation potential method according to the formulation of Foucrault et al. (1992) which reproduced very well the atomic lithium spectral properties. They found excellent agreement between experimental data and the various spectroscopic constants and for the vibrational levels of the various electronic states. A further analysis of the ground and excited states of this molecule and of the corresponding dipole functions was recently done using the bonded tableau unitary group approach (Mo et al. 1993) and provided a clear physical explanation for the dipole moment behaviour in these systems as a function of internuclear distance.

Fig. 6. Computed transition moments of rovibrational processes for $v=0 \rightarrow v^{\prime}=1$ and involving all possible rotational states. The lower part shows the calculations as functions of initial $J$ values, while the upper part refers to transitions as functions of frequency. In each part, the lower curve refers to $\Delta J=+1$ transitions, while the upper curve refers to the $\Delta J=-1$ transitions. The two curves cross at $J=70$ and at $1200 \mathrm{~cm}^{-1}$

More extensive calculations were recently repeated using the pseudopotential approach of Boutalib and Gadea and generating the lowest eight diabatic and adiabatic states over a very broad range of internuclear distances (Berriche 1995). A denser set of points was employed to evaluate the full potential energy curves and the ground electronic state was carefully analysed over a broad range of distances. A further correction due to the variation of the electron affinity of the $\mathrm{X}^{1} \Sigma^{+}$state as a function of internuclear distance was also introduced for the $\mathrm{H}$ atom, in order to take into account the basis set superposition effects from the lithium atom (Berriche 1995). A comparison of various constants computed for the ground electronic state with experiments and with other calculations 

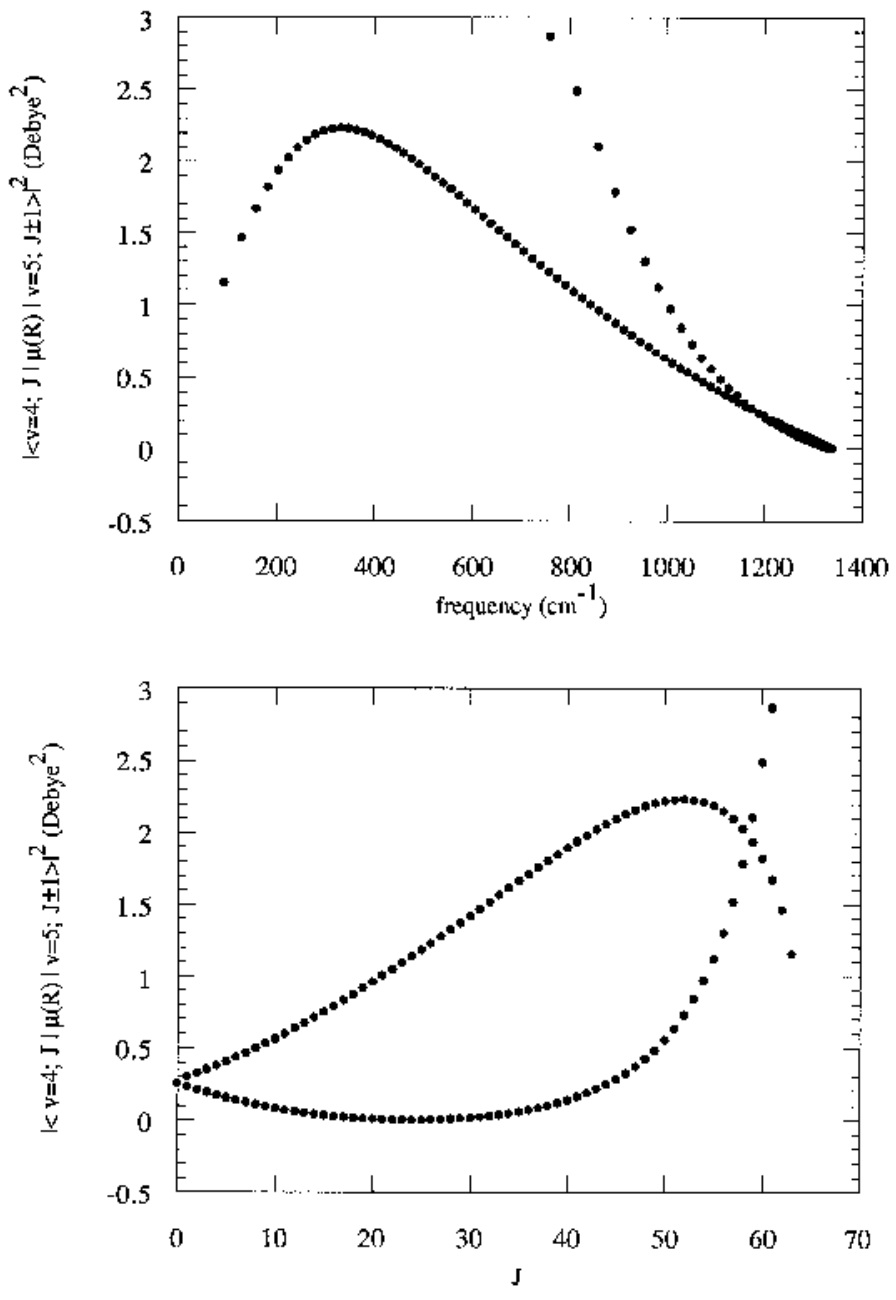

Fig. 7. Same as in Fig. 6 but for rovibrational transitions between $v=4$ and $v^{\prime}=5$. The meaning of all curves of all symbols are the same as in Fig. 6

is shown in Table 1. One clearly sees there that the calculated properties from the present potential energy curves (PEC's) are indeed in good accord with the experimental findings and compare very well with other existing calculations. It is interesting to further note that earlier, very accurate, calculations (Klopper et al. 1991) determined the binding energy of the ground electronic state of $\mathrm{LiH}$ with respect to the $\mathrm{Li}^{+}+\mathrm{H}^{-}$asymptotic limit, using perturbative Moeller-Plesset calculations with all electrons of the system. Using two different corrections they found it to be either $7.217 \mathrm{eV}$ or $7.211 \mathrm{eV}$. Our present result $(7.158 \mathrm{eV})$ is even closer to the experimental value of $7.151 \mathrm{eV}$ and within its stated experimental error (Huber et al. 1978).

In Table 2 we report the vibrational levels for the $\mathrm{X}^{1} \Sigma^{+}$state and the energy spacings among levels. It is interesting to note that the earlier results which used essentially the same method but employed a less extended basis set (Boutalib \& Gadea 1992) found 23 bound levels, in accord with the previous calculations of P \& L. Recent calculations of the bound states using modification of the PEC of P \& L found a total of 22 levels (Dalgarno et al. 1995) which were agreeing to within $10 \mathrm{~cm}^{-1}$ with the observed values. Our computed points were 110 within the range of internuclear distances between $R_{\min }=1.8 \mathrm{a}$.u. and $R_{\max }=243$ a.u.. The numerical solutions for the bound wavefunctions was found following a numerical procedure already discussed in our previous work (Gianturco \& Gallese 1988; Gianturco et al. 1989). In the present case, the new potential energy curve supports 24 vibrational levels, one more than those predicted before, as expected by the increase in the well depth shown in Table 1. It is interesting to note that the hybrid potential curve of Zemke \& Stwalley (Zemke \& Stwalley 1980) also found 24 bound states. The present spacings between levels are compared with the calculations of $\mathrm{P} \& \mathrm{~L}$ in Table 2 , where one sees that our more strongly bound levels (up to $v=15$ ) differ from theirs by at most $10 \mathrm{~cm}^{-1}$ and present very similar transition energy values. The six top transitions, as expected, differ by increasing amounts up to $\sim 50 \mathrm{~cm}^{-1}$ due to the different treatment of the long-range part of the interaction between our present calculations and those of $\mathrm{P}$ $\& \mathrm{~L}$. The comparison with the available experimental values, on the other hand, suggests better agreement of our transition energies with the values from Way et al. (Way et al. 1973) than that shown by the calculations of P \& L, as we move towards the higher bound levels of our computed PEC. Furthermore, the comparison with the more recent recommended potential curves of Chan et al. (Chan et al. 1986) indicates a remarkable good agreement with our higher levels $(v>15)$, better than that from the earlier calculations of P \& L as it was quoted by Zemke \& Stwalley (Zemke \& Stwalley 1980). As expected, an extra bound level produces one additional transition $\left(E_{23}-E_{22}\right)$ which is not shown by the experimentally recommended values of Chan et al. (Chan et al. 1986). On the whole, however, the calculated level positions and the zero-point energy value (ZPE) given by our PEC are in rather good accord with the most recently reported experimental analysis (Stwalley et al. 1993). A qualitative display of the full curve for the $\mathrm{X}^{1} \Sigma^{+}$state is presented in Fig. 1, where one clearly sees the well region (below) and the long-range part of the attractive interaction (above). A further interesting test of the quality of our computed energy curve could be gleaned from a comparison of existing experiments of the RV levels in $\mathrm{LiH}$ with those produced with our present calculations. Recent, accurate measurements (Bellini et al. 1994) have reported, in fact, rotational transitions between the lowerlying vibrational levels of the $\mathrm{X}^{1} \Sigma^{+}$electronic state of $\mathrm{LiH}$. We have carried out the corresponding calculations using the procedure mentioned before (Gianturco et al. 1989) and the results are compared in Table 3 . It is indeed reassuring to see that the overall error remains consistently below $1 \%$ over the whole range of available results. 
In conclusion, the tests of the various computed properties from our potential energy curve suggest that, for the ground electronic state, we can consider the present calculation as having produced one of the best available descriptions of the RV bound states for the $\mathrm{LiH}$ molecule.

\subsection{The dipole moment function}

Experimental and theoretical values of the dipole moments, the radial dipole functions and the dipole moment derivatives have been reported by many workers over the years and the relevant literature has recently been reviewed in great detail (Stwalley et al. 1993).

In Table 4 we compare our computed dipole function with that calculated by $\mathrm{P} \& \mathrm{~L}$. The latter was given between $R=1.75$ a.u. and up to 17.5 a.u. and was in good agreement with the CI calculations of D \& $\mathrm{H}$ and to within $0.3 \%$ of the more recent calculations of Ogilvie and coworkers (Ogilvie et al. 1994) that provide estimates for $R$ between 2.215 a.u. and 4.115 a.u.. A recent application of the above calculations (Dalgarno et al. 1995) further extends the dipole function beyond 17.5 a.u. by using the long range form $d_{7} / R^{7}$ with the coefficient obtained by fitting the ab-initio points of the outer range of radial values. Our computed $\mu(R)$ was obtained between 1.80 a.u. and 20.0 a.u. and fitted with cubic spline functions within this range of values. Its general shape is shown in Fig. 2.

It is interesting to see that our calculations follow very closely the earlier results of $\mathrm{D} \& \mathrm{H}$, which were recently employed in calculating radiative ricombination rates (Dalgarno et al. 1995), as we shall further discuss below.

We have also calculated the expectation values of the dipole moment function for all the bound vibrational levels of the $\mathrm{X}^{1} \Sigma^{+}$state and for the $J=0$ situation, i.e. for pure vibrational wavefunctions. These quantities were also computed by Zemke \& Stwalley (Zemke \& Stwalley 1980) using the dipole function of D \& $\mathrm{H}$ and the PEC discussed in their work. In their case, the number of bound vibrational states was 24 , as in our present potential curve, and therefore we can make a comparison with all their values as we show in Table 5, where some experimental quantities are also listed (Wharton et al. 1960; Rothstein 1969).

For the higher vibrational levels a broader range of internuclear distances must be used because of the slow decay with increasing distance of the bound wavefunctions near the dissociation limit in the classically forbidden region, while lower $v$ levels obviously converge at smaller $R_{\max }$ values. The computed dipole moment expectation values of Table 5 show an initial slow increase with $v$, since $\langle R\rangle$ increases and $\langle\mu\rangle$ decreases by increasing the vibrational quantum number, while they change drastically as the dissociation limit is approached. This is due to the fact that $\mu$ drops to zero at large $\langle R\rangle$ values and therefore the corresponding expectation value decreases very dramatically when going from $v=21$ to $v=23$, both in the present calculations and in those of Zemke \& Stwalley (Zemke \& Stwalley 1980). As we shall discuss below, such behaviour is clearly going to affect the results of the radiative emission lines.

\subsection{Radiative transition probabilities}

There are four basic kinds of spectroscopic intensity measurements, depending on whether absorption or emission is involved and whether photons or energy flux is detected. In each case, however, the absolute intensities involve a power of the transition frequency $\nu_{v^{\prime} J^{\prime}, v^{\prime \prime} J^{\prime \prime}}$, where

$$
\nu_{v^{\prime} J^{\prime}, v^{\prime \prime} J^{\prime \prime}}=\frac{1}{h}\left(E_{v^{\prime} J^{\prime}}-E_{v^{\prime \prime} J^{\prime \prime}}\right)
$$

multiplied by the square of a dipole moment matrix element

$$
\mu_{v^{\prime} J^{\prime}, v^{\prime \prime} J^{\prime \prime}}=\int_{0}^{+\infty} \Psi_{v^{\prime} J^{\prime}}^{*}(R) \mu(R) \Psi_{v^{\prime \prime} J^{\prime \prime}}(R) \mathrm{d} R
$$

with normalized initial and final wavefunctions and with the dipole moment function discussed before. Note that, if $v^{\prime}=v^{\prime \prime}$ and $J^{\prime}=J^{\prime \prime}=0$, we simply recover the dipole moment expectation value $\langle\mu\rangle$ in the $v^{\prime}$ vibrational level and without rotational effects, as shown by the present calculations in Table 5 .

For an absorption measured by the observed decrease in photon flux the transition probability is proportional to $|\mu|^{2}$ of Eq. (7) and has no frequency dependence. It is commonly expressed by the line strength of the process or by its Einstein $B$ coefficient. For an absorption measured by a decrease in energy flux, then the transition probability is proportional to $|\mu|^{2} \nu$. In this case, the oscillator strength $f$ or the absorption coefficient $\alpha$ are commonly used. For emission studies, the corresponding transition probabilities go as $|\mu|^{2} \nu^{3}$ and as $|\mu|^{2} \nu^{4}$ for photons and energy detectors, respectively (Wiese et al. 1966). Thus, astronomical observations will be greatly affected by both the value of the transition moments and that of the transition frequency, as we shall further discuss below. The various Einstein coefficients are related to the spontaneous photon emission intensities and each of the contributions comes from the transition probabilities of emission studies, $A_{v^{\prime} J^{\prime}, v^{\prime \prime} J^{\prime \prime}}\left(\right.$ in s $^{-1}$ ) where the $\left|v^{\prime} J^{\prime}\right\rangle$ is the upper level and $\left|v^{\prime \prime} J^{\prime \prime}\right\rangle$ the lower one. The total coefficient arising from all the bound $\rightarrow$ bound transitions of the $\mathrm{X}^{1} \Sigma^{+} \rightarrow \mathrm{X}^{1} \Sigma^{+}$ band system may be simply obtained from the expression:

$$
\begin{aligned}
& A_{v^{\prime} J^{\prime}}=\frac{1}{2 J^{\prime}+1} \\
& \left\{\left(J^{\prime}\right) \sum_{v^{\prime \prime}=0}^{v^{\prime}} A_{v^{\prime} J^{\prime}, v^{\prime \prime} J^{\prime}-1}+\left(J^{\prime}+1\right) \sum_{\substack{v^{\prime \prime}=0 \\
v^{\prime}=1}} A_{v^{\prime} J^{\prime}, v^{\prime \prime} J^{\prime}+1}\right\}
\end{aligned}
$$




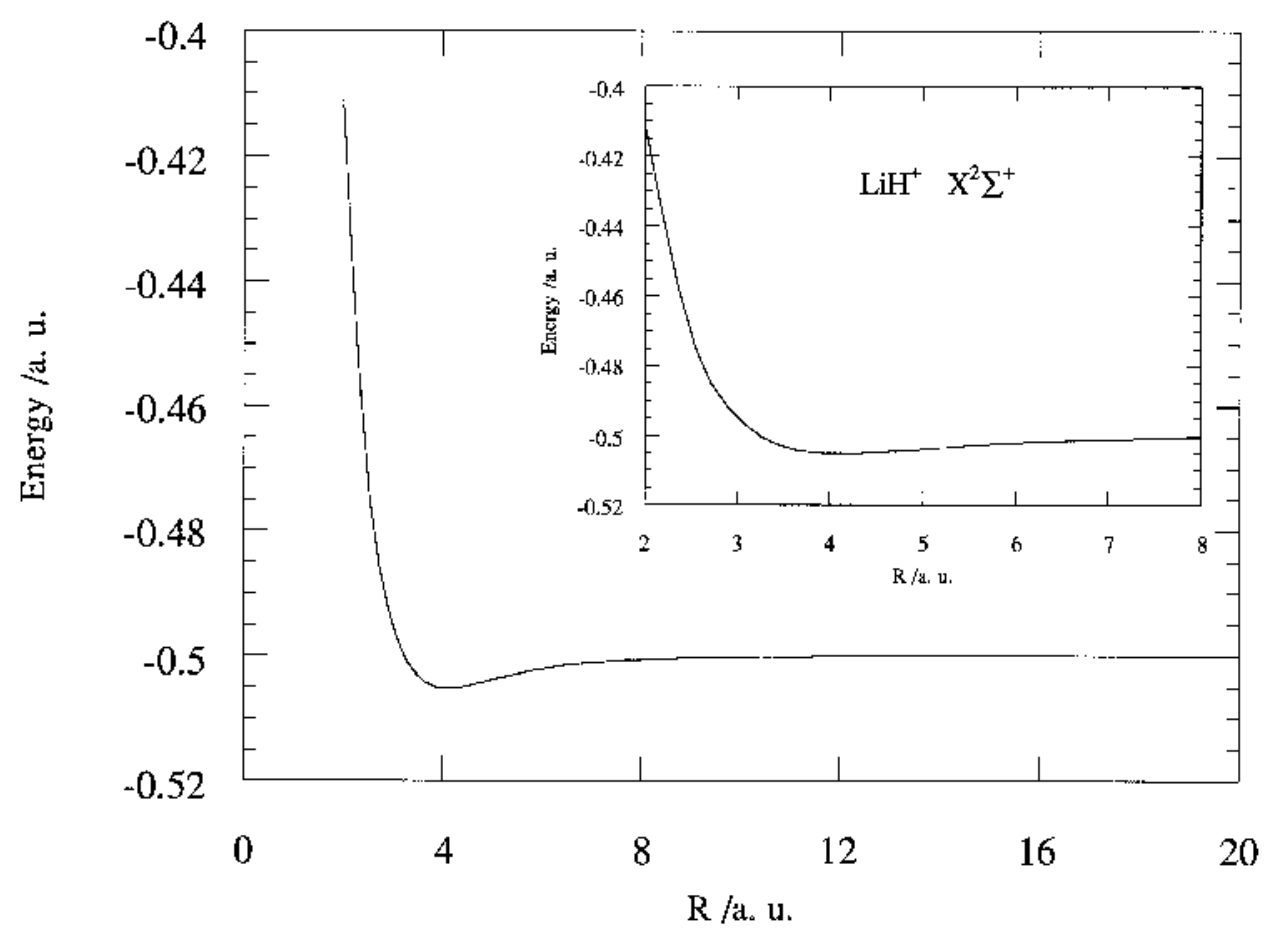

Fig. 8. Computed ground electronic state for the $\mathrm{X}^{2} \Sigma^{+}$electronic state of the $\mathrm{LiH}^{+}$molecule. The inset shows the well region and the onset the repulsive wall. All quantities in atomic units

The reciprocal of these coefficients values give the radiative lifetimes $\tau_{v^{\prime} J^{\prime}}$ which correspond to the spontaneous radiative lifetimes controlling the rate of emission from the various molecular levels.

An example of the behaviour of squared values of the transition moments for the case of $J^{\prime}=J^{\prime \prime}=0$ is shown in Fig. 3, where we report the results of our calculations as a function of the initial vibrational level (top) and of the absorption frequency of each line (bottom). The three curves refer to the dipole allowed fundamental transition $(\Delta v=1)$ and two further examples with larger quantum jumps $(\Delta v=2$ and 3$)$. One clearly sees there that, as one moves to higher initial states, the $\Delta v=1$ transitions become less intense than the $\Delta v=2$ transitions, while recovering their dominant role from transitions from $v>18$. This is in keeping with the earlier calculations (Stwalley et al. 1993) and indicates the large anharmonicity of the higher bound levels in this system. In terms of energy ranges involved in the absorption (or emission) process, we note that the most intense band is appearing at around $300 \mathrm{~cm}^{-1}$, while the second region of intense transitions appears between 1000 and $1500 \mathrm{~cm}^{-1}$.

As one considers the pure rotational transitions, it is of some interest to examine separately those involving the lowest vibrational bound state of the $\mathrm{LiH}$ molecule from those occurring from excited vibrational levels. The calculations reported in Fig. 4 indicate the computed intensity values as a function of frequency and also show (in the inset) their behaviour as a function of final rotational quantum number $J$. The latter quantum number goes up to 76 within that level, the states with $J \geq 61$ turn out to be quasi-bound states, the intensity of the transitions increases with $J$, showing its maximum value for $J=65$. The corresponding energy dependence therefore indicates the most intense transitions as being located in the range of 350 to $400 \mathrm{~cm}^{-1}$, i.e. rather close to the most intense lines for pure vibrational transitions. This is a useful piece of information if observational selections of intensity windows need to be performed in either the laboratory or the astronomical measurements (De Bernardis et al. 1993; Bellini et al. 1994).

The pure rotational transitions were further computed for all possible rotational states and a set of representative intensities is reported in Fig. 5, both as a function of the transition frequency (top) and of the initial $J$ value for a few vibrational levels. All calculations were carried out by numerically generating the rotovibrational levels of the $X$ potential energy curve and further evaluating the transition moments by quadrature over the computed $\mu(R)$ behaviour, as discussed before. Numerical convergence was carefully tested by increasing the range of radial values employed and by varying the adaptive grid of integration points along it.

The transition intensities as functions of $J$ indicate that they steadily decrease as the rotational initial quantum number decreases and as the vibrational index 
increases up to $v \sim 10$, while the trend is reversed for higher $v$ values, since we observe that from $v \geq 11$ the most intense transitions involve the lower $J$ values. Thus, one sees from the present calculations that the most intense absorption (or emission) should involve the lowest bound levels for vibrations and, possibly, rotationally 'hot' molecular states. Furthermore, the results shown on the top part of the same figure indicate that the next most intense transitions would occur for pure rotational levels with low $J$ but involving 'hot' vibrational levels around $v \sim 11$. This set of transitions is shown, by the upper part of Fig. 5, to occur between 250 and $400 \mathrm{~cm}^{-1}$, i.e. fairly close to the previous range of energies. Thus, the pure vibrational and pure rotational transitions indicate, from the present calculations, that the most intense lines should be located around the energy range of $400 \mathrm{~cm}^{-1}$.

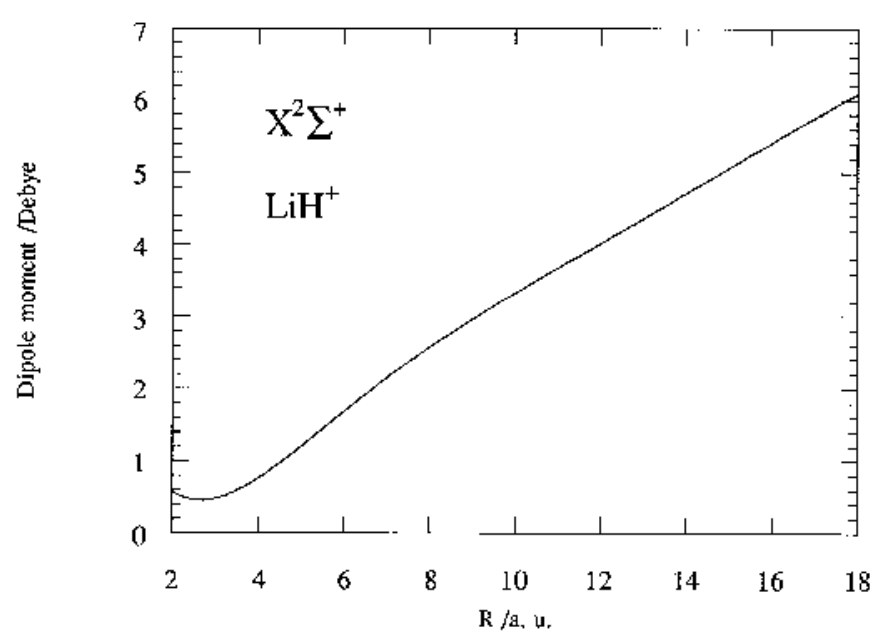

Fig. 9. Computed dipole function for the $\mathrm{X}^{2} \Sigma^{+}$electronic state of the $\mathrm{LiH}$ ion. The distance is given in atomic units and the $\mu$ values in Debye

Finally, the full range of rovibrational transitions and transition intensities has been computed for the present system and some representative results are shown in Figs. 6 and 7.

The curves in Fig. 6 report absorption transitions from the ground vibrational level to the first vibrational level $(\Delta v=1)$ and with different changes in the rotational indices. The lower part of the figure shows such transitions as a function of initial $J$ value: the curve with lower intensities at low $J$ refers to $\Delta J=+1$ transitions, while the curve with increasing and higher intensities refers to the $\Delta J=-1$ transitions. Due to the high anharmonicity of the potential, however, one sees that, at $J=70$, the trend is inverted and the few higher transitions with $\Delta J=-1$ become less intense than those with $\Delta J=+1$ and involving high $J$ values. Correspondingly, the energy-dependent behaviour shown in the upper part of the same figure indicates that there are two types of transitions with large intensity values: those around 200 to $400 \mathrm{~cm}^{-1}$ which correspond to $\Delta J=-1$ transitions for $J$ values between 65 and 55 and those around 800 to $900 \mathrm{~cm}^{-1}$ that correspond to $\Delta J=+1$ for $J$ values around 70 . In other words, possible frequency regions of observation are suggested at rotational temperatures that are rather high for the neutral molecule but involve again its lowest vibrational level.

This pattern of behaviour is kept largely unchanged as we observe transitions between higher vibrational levels and a specific example is shown in Fig. 7, where the calculations are essentially the same as those of Fig. 6 but now involve the $\Delta v=1$ transition between the $v=4$ and the $v=5$ levels. As one clearly sees, the pattern is very similar, the only change being that fewer $J$ values are possible and the crossing between curves occurs now at a lower value of the excited rotational level. In terms of energy range, the most intense transitions still appear either around $400 \mathrm{~cm}^{-1}$ and around $800 \mathrm{~cm}^{-1}$, as before. As one goes up with the initial $v$ value involved, the range of accessible $J$ values obviously decreases, but the overall dependence of intensity and frequency over the range of such values remains largely unchanged.

Calculations were carried out for all the transitions between all vibrational levels and all the possible rotational quantum numbers. The actual tables with all the relevant computed values are available on request.

\section{Rovibrational transitions in $\mathrm{LiH}^{+}$ions}

\subsection{The ground state adiabatic potential}

Several calculations have been carried out over the years for the equilibrium geometry, and its related properties, of the lithium hidride positive ion (Stwalley \& Zemke 1993; Morgan et al. 1985). This is a weakly bound ionic molecule with $D e$ values currently converging at $\sim 1100 \mathrm{~cm}^{-1}$ and therefore supporting a much smaller number of bound states than in the case of its neutral counterpart.

The full potential energy curves for its lowest-lying electron state $\left(\mathrm{X}^{2} \Sigma^{+}\right)$and for the next low-lying states (five $\Sigma$ states and two $\Pi$ states) have been already presented elsewhere (Berriche \& Gadea 1995). The two lowest separated atom limits for $\mathrm{LiH}^{+}$are given by $\mathrm{Li}^{+}\left({ }^{1} \mathrm{~S}\right)+$ $\mathrm{H}\left({ }^{2} \mathrm{~S}\right)$ and by $\mathrm{Li}\left({ }^{2} \mathrm{~S}\right)+\mathrm{H}^{+}$. Each of them correlates to a single molecular state of ${ }^{2} \Sigma^{+}$symmetry but they are separated by the difference in ionisation potentals between the two partners $(8.214 \mathrm{eV})$. The calculations involving a non-empirical pseudopotential for the $\operatorname{Li}\left(1 \mathrm{~s}^{2}\right)$ core and accurate core-valence correlation corrections (Berriche \& Gadea 1995) produced a slightly deeper and wider well than the earlier MRD-CI calculations on the same system and for both the $\mathrm{X}^{2} \Sigma^{+}$and $2^{2} \Sigma^{+}$electronic states (Voitik et al. 1970). We have employed the former to calculate the rovibrational levels and the latter to evaluate the transition moments discussed below. 

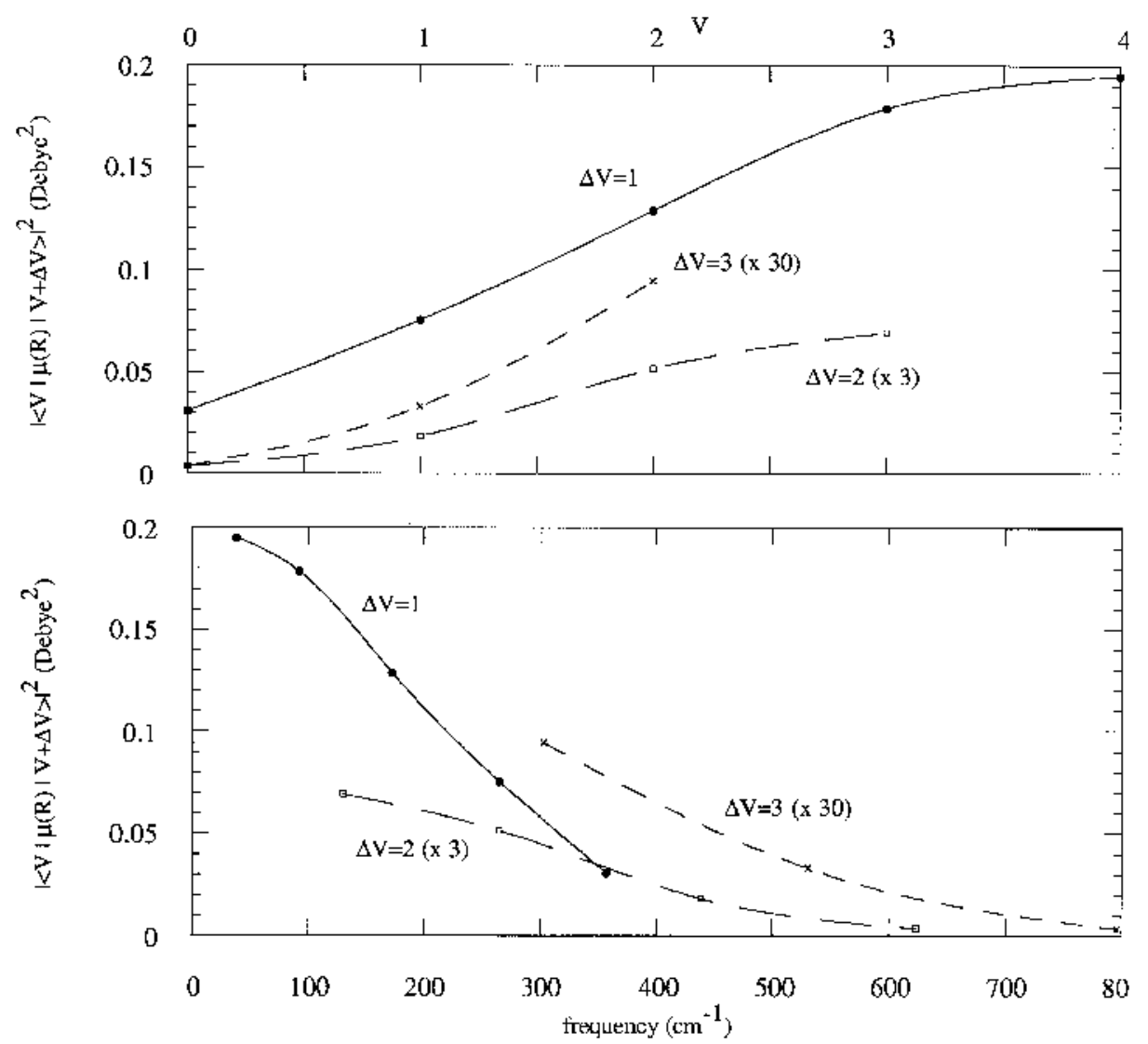

Fig. 10. Pure vibrational transitions for the ground electronic state of $\mathrm{LiH}^{+}$. Top curves are given as a function of vibrational index $v$, while the lower curves are plotted as a function of frequency

Figure 8 reports the general shape of the $\mathrm{X}^{2} \Sigma^{+}$lower state, with the inset showing the well region and the onset of the repulsive wall. The equilibrium distance at the minimum turns out to be 4.15 a.u., with a well depth of $0.13 \mathrm{eV}$. These results are in good accord with earlier pseudopotential calculations (Berriche \& Gadea 1995) and with recent calculations using a full CI within the space of one-electron functions (Grabandt et al. 1992).

The bound vibrational states were computed as before and we found six of them for the $\mathrm{X}^{2} \Sigma^{+}$potential. Table 6 presents a comparison of the present calculations with the earlier results (Grabandt et al. 1992; Tortorelli et al. 1978; Dyer \& Steiner 1986). We see that most calculations suggest the same total number of bound states and they agree reasonably well with each other. The excited $2^{2} \Sigma^{+}$state dissociating to the charge-exchange asymptotic atoms turns out to be more strongly bound and the corresponding computed PEC's support either 16 vibrational levels (Voitik et al. 1970) or 28 levels (Berriche \& Gadea 1995).

\subsection{The dipole moment function}

The dipole moment function computed from Voitik et al. (Voitik et al. 1970) for the ground electronic state is shown in Fig. 9 over the range of available points, while the spline function used to fit them and to extrapolate to higher radial values is also shown as a solid line. All $\mu(R)$ values are given in units of Debye and the dipole moment is referred to the lithium atom as a reference origin. At the equilibrium geometry of 4.15 a.u. its value is 0.250 a.u., which is reasonably close to the 0.2305 a.u. calculated value of Dyer \& Steiner (Dyer \& Steiner 1986) for an equlibrium geometry of 4.25 a.u..

\subsection{Computed transition moments}

The transition moments calculated for the pure vibrational processes are shown in Fig. 10, where the results from single excitations $(\Delta v=1)$ and multiple excitations $(\Delta v=2$ and 3$)$ are reported as a function of initial level (top) and of transition frequency (bottom). Contrary to what was shown by the same quantities for the neutral molecule (see Fig. 3 before) the single quantum transitions are always more intense than the multiple quantum jumps, no matter which initial level is considered. The smaller 


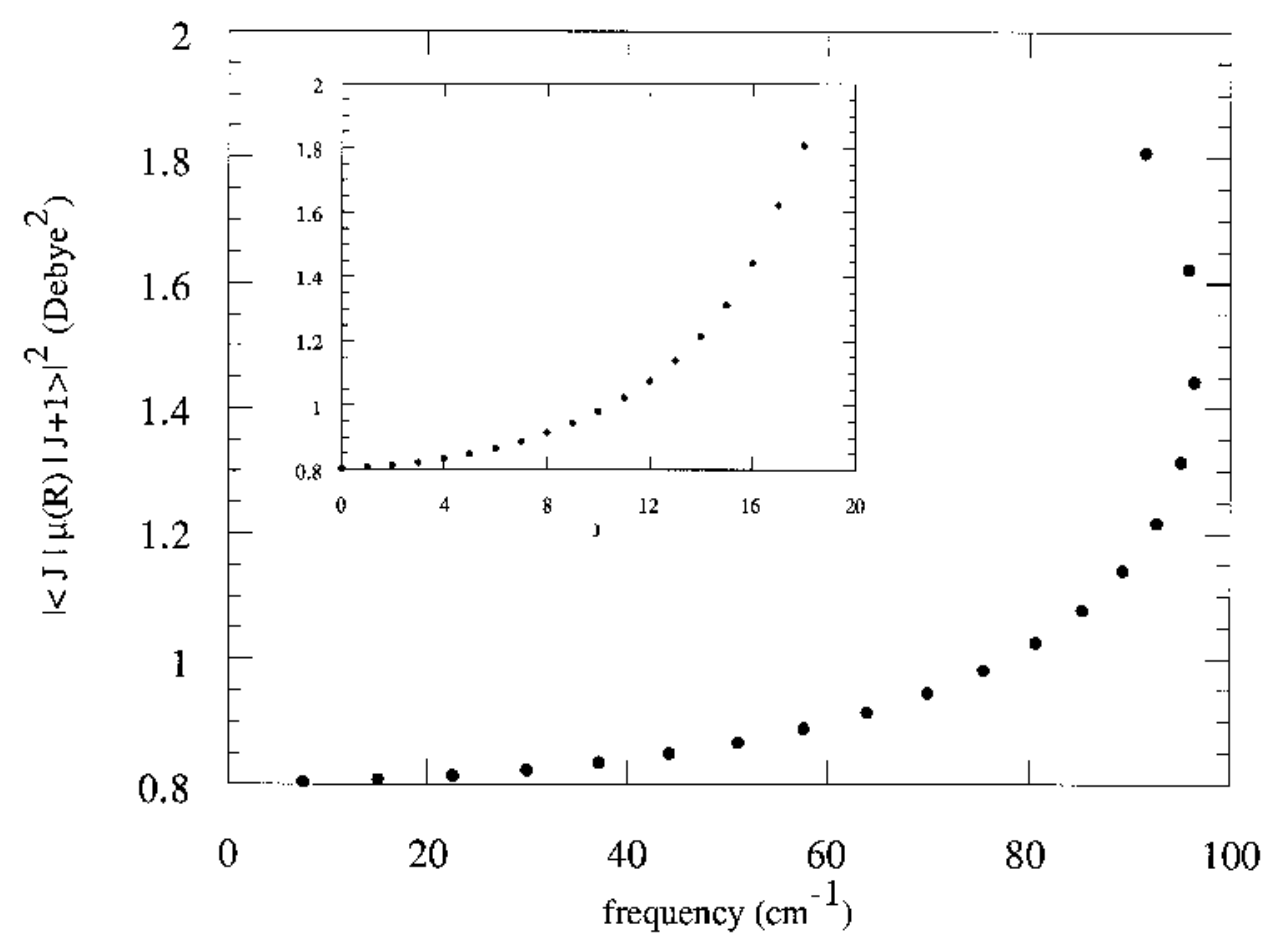

Fig. 11. Computed pure rotational transitions in the LiH ion for the ground vibrational state as a function of frequency and using the $\mathrm{X}^{2} \Sigma^{+}$potential. Inset: intensity of transition as a function of initial $J$ value

Table 6. Computed vibrational level spacings for the $\mathrm{X}^{2} \Sigma^{+}$electronic state of $\mathrm{LiH}^{+}$. All data in $\mathrm{cm}^{-1}$

\begin{tabular}{|c|c|c|c|c|c|}
\hline \multirow[t]{2}{*}{$\mathrm{v}$} & $\mathrm{E}_{\gamma^{-}} \mathrm{E}_{\gamma-1}$ & $E_{v}-E_{v-1}$ & $\mathrm{E}_{\mathrm{v}}-\mathrm{E}_{\mathrm{\psi}-1}$ & $E_{\gamma}-E_{\psi-1}$ & $E_{\psi}-E_{\psi-1}$ \\
\hline & present work & a & b & $c$ & d \\
\hline $\begin{array}{l}1 \\
2 \\
3 \\
4 \\
5 \\
6\end{array}$ & $\begin{array}{l}357.43 \\
265.61 \\
172.96 \\
92.34 \\
38.16 \\
-\end{array}$ & $\begin{array}{l}357.43 \\
265.61 \\
172.96 \\
92.90 \\
37.45 \\
9.22\end{array}$ & $\begin{array}{l}354.09 \\
261.63 \\
169.48 \\
90.311 \\
35.585 \\
-\end{array}$ & $\begin{array}{l}335 \\
245 \\
152 \\
- \\
- \\
-\end{array}$ & $\begin{array}{l}313.85 \\
245.81 \\
175.58 \\
105.35 \\
37.310 \\
-\quad\end{array}$ \\
\hline
\end{tabular}

a) Berriche \& Gadea 1995; b) Dalgarno et al. 1995 ; c) Grabandt et al. 1992; d) Dyer \& Steiner 1986

number of bound levels in the present instance and the shallower potential well of the ionic molecule suggest that the anharmonicity effect plays here a less prominent role, while the changes in the spatial distribution of the excited vibrational levels invariably cause a lowering of the transition moment values. As a result, even the most intense transitions for the present system are really markedly weaker than those for the neutral molecule and appear at lower frequencies (i.e. between 50 and $100 \mathrm{~cm}^{-1}$ ), thus making their observations in emission mode much harder to obtain.

The corresponding behaviour of purely rotational transitions is shown in Fig. 11 for the levels pertaining to the $v=0$ bound vibrational state. Due to the nature of the in- teraction, the number of rovibrational levels is now much smaller than in the case of the neutral counterpart: $J_{\max }$ is here equal to 19 for $v=0$ (the states with $J=17$, 18 and 19 are quasi-bound levels) and the total number of RV levels is equal to 64 , in agreement with the recent results of Dalgarno et al. (Dalgarno et al. 1995).

At variance with the behaviour exhibited by the neutral system (see Fig. 4), the intensity of the pure rotational transition increases with the increasing $J$ value, without going through an intensity maximum for one of the higher rotational quantum numbers, as it occurs in Fig. 4. Furthermore, one sees that the frequencies of these transitions increase with increasing $J$ and show a frequency lowering effect at higher $J$ that is less marked than in the case of 

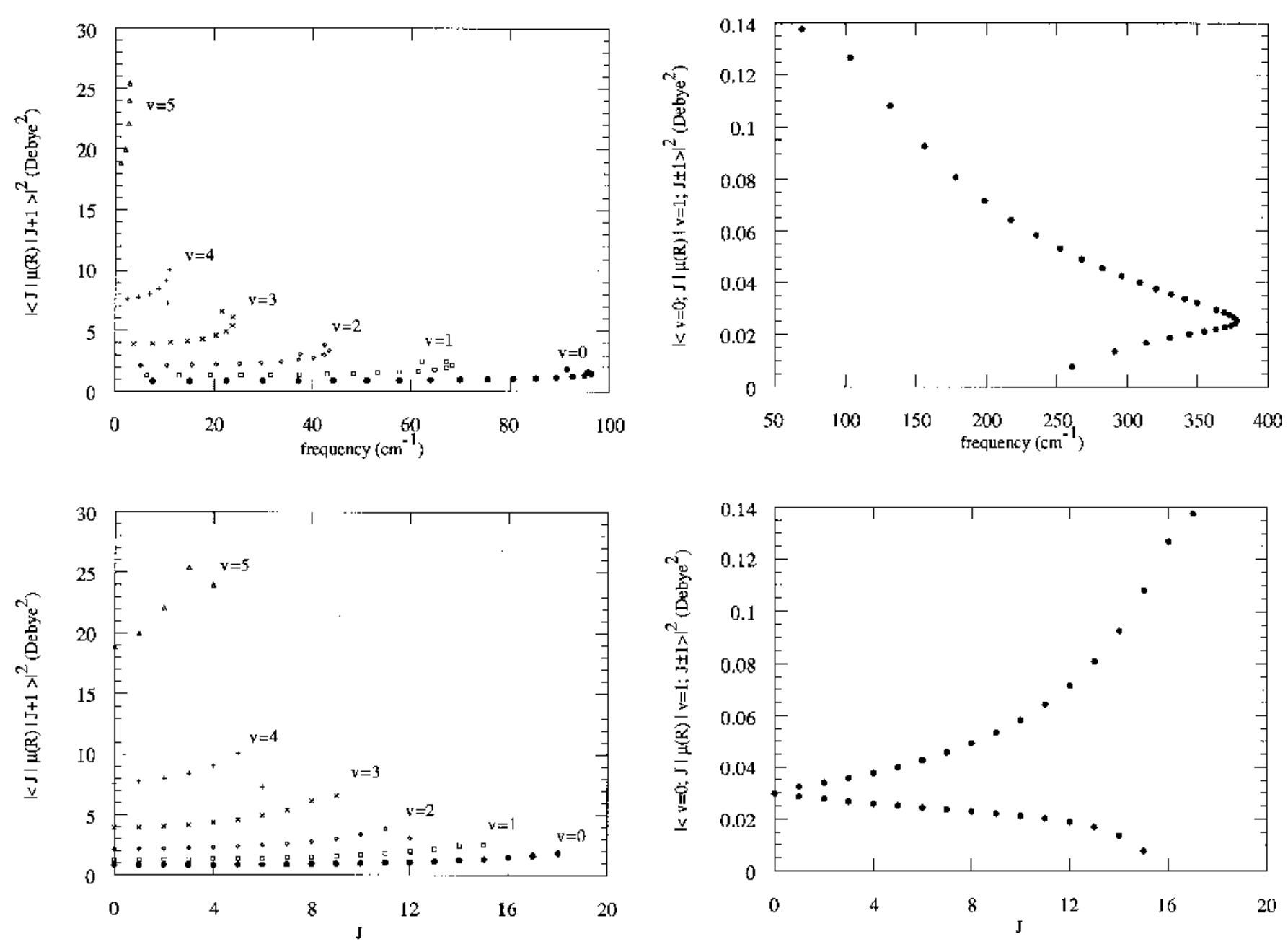

Fig. 12. Pure rotational excitations in the $\mathrm{LiH}^{+}$molecule as a function of vibrational state. Top: frequency range. Bottom: intensity as a function of initial $J$ value

Fig. 13. Computed rotovibrational transitions for the $\mathrm{LiH}$ ion as a function of frequency (top) and initial rotational state (bottom). The vibrational transition is the $v=0 \rightarrow v^{\prime}=1$

the neutral molecule. Thus, the most intense lines here are also those appearing in the highest frequency range around $90 \mathrm{~cm}^{-1}$. Their intensities, however, are nearly a factor of fifty smaller than in the case of the neutral molecule.

An interesting trend of transition intensities as function of the initial vibrational state is shown by the calculations displayed in Fig. 12, where we have reported the pure rotational transitions originating within each of the six vibrational states of the $\mathrm{X}^{2} \Sigma^{+}$curve. One clearly sees in that figure how the intensities of the transitions increase with increasing vibrational state and, by the time the $v=5$ state is reached, the intensities have increased more than a factor of 20 . On the other hand, the intensity behaviour within each rotational manifold appears to be different depending on the vibrational state: for the more bound vibrational levels the intensity of the rotational transitions increases monotonically with $J$, while for some of the higher $v$ states $(v=2,4$ and 5$)$ the intensity goes

through a maximum. Likewise, the frequency dependence of all the above transitions shows, within each manifold, an intensity maximum with a lowering of the associated frequencies, as seen much more markedly in Fig. 5 for the neutral molecule. The latter system, however, exhibited a lowering of the intensities and frequencies of transitions as $v$ increased, due to the expected spatial 'spreading' of the higher vibrational wavefunctions with respect to the limited range of action of the dipole operator.

The full range of rovibrational transitions associated with the $\mathrm{X}^{2} \Sigma^{+}$state of the ionic molecule is shown in Figs. 13 and 14, to be compared with the behaviour of the larger number of transitions for the neutral molecule already reported in Figs. 6 and 7. A number of differences can be gleaned from examining the figures for the $\mathrm{LiH}^{+}$ molecule: 

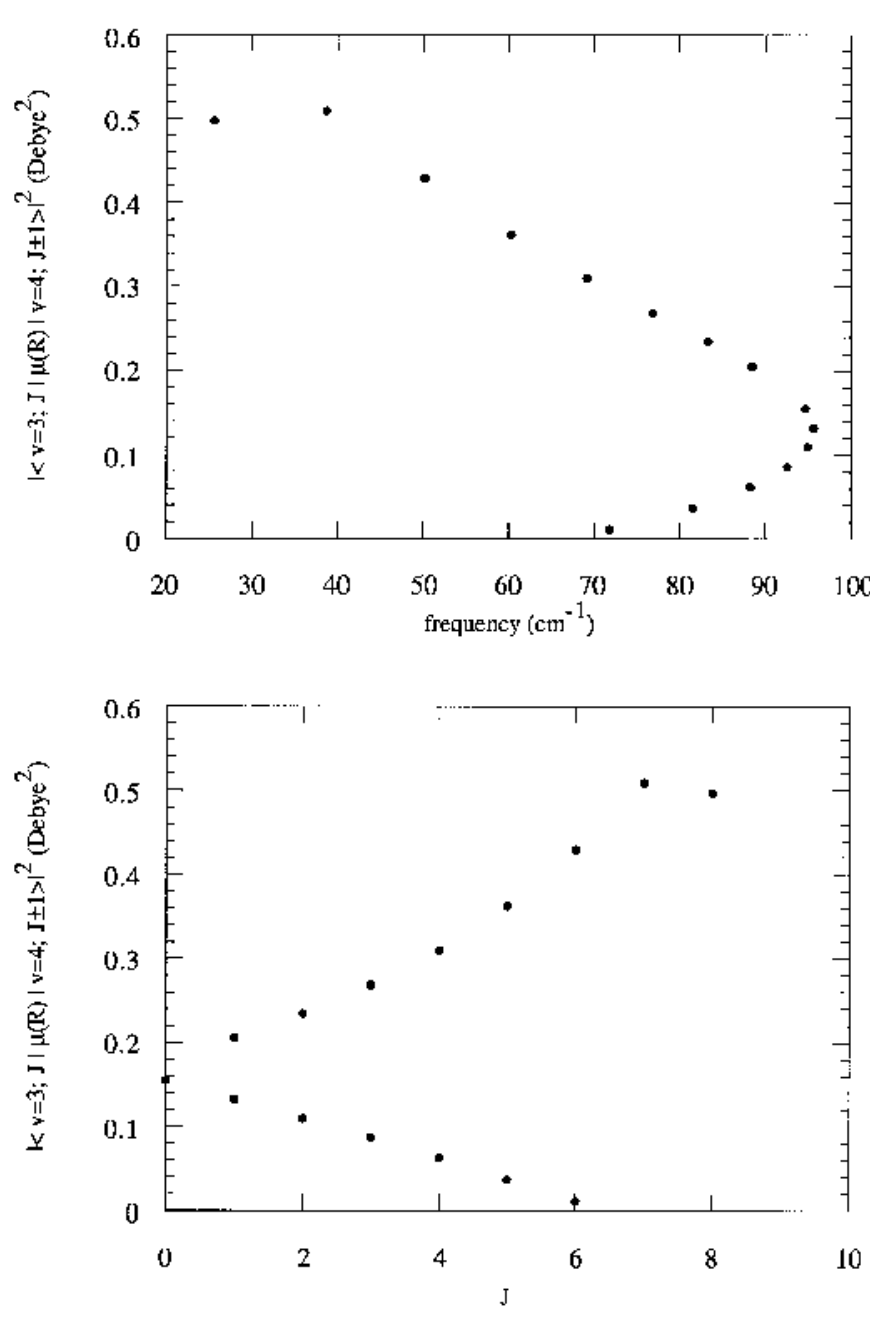

Fig. 14. Same as in Fig. 13 but for the vibrational transition between the $v=3$ and $v^{\prime}=4$ levels

i) the overall intensities of the transitions involving the lowest vibrational states are much weaker for the ionic molecule than they are for the neutral target;

ii) the intensities increase markedly with increasing the $v$ quantum numbers involved (see Fig. 14) but not nearly as much as in the neutral case, which still scales by a factor of about five more intense (see Fig. 7);

iii) the transitions with $\Delta J=-1$ are invariably more intense than those with $\Delta J=+1$ and remain so across the whole range of $J$ values;

iv) the difference in frequency between the above sets of transitions increases as $J$ increases and the most intense transitions correspond to the highest $J$ in each manifold.

The general conclusion from the behaviour of the rotovibrational transitions in the ionic molecule is that the most intense lines will be much less intense than in the case of $\mathrm{LiH}$ and will appear in the range of frequencies around $100 \mathrm{~cm}^{-1}$. They will correspond to rotationally and vibrationally 'hot' molecular RV states, while the transitions between the lower-lying states will be appearing at lower frequencies and will be markedly weaker.

\section{Conclusions}

In this work we have examined in detail the behaviour of the RV transitions for the ground electronic states of the $\mathrm{LiH}\left(\mathrm{X}^{1} \Sigma^{+}\right)$and $\mathrm{LiH}^{+}\left(\mathrm{X}^{2} \Sigma^{+}\right)$molecules, using very accurate potential energy curves and the corresponding calculated dipole functions. We have compared some of the general features of the two molecules with the existing experiments and with earlier calculations of the same properties and found rather good agreement between the present results and the earlier findings on both systems.

The full range of bound states, vibrational and rotational states, is also examined and compares favourably with the most recent calculations. The general behaviour of the intensities is such that one can select possible ranges of energy which would correspond to the most intense absorption or emission lines in both systems. The ionic species is invariably found to yield less intense dipole transitions and its most intense lines appear at much lower frequencies. Such differences should, therefore, have quite a marked consequence on the corresponding radiative recombination rates and on observing the possible presence of such primordial molecules in the post-recombination universe. These calculations should make possible for one to quantitatively assess where in the observational frequency range the most intense lines will appear and which ones will be the more likely to be observed.

The actual calculations for the associated radiative recombination cross sections and the cooling functions that stem from the above analysis are currently under way and will be presented elsewhere. The present results should be of use in selecting specific observational windows, which would in turn make most likely a possible detection of both species in order to assess their importance in modelling the primordial cloud formation discussed in the Introduction.

Acknowledgements. The financial support of the Italian National Research Council (CNR) and of the University of Rome Research Grants Committee is gratefully acknowledged. (H.B.) thanks the University of Rome for supporting a visiting stage when this work was begun. (F.A.G.) thanks the Institute of Atomic and Molecular Physics (ITAMP) at Harvard University for a Visiting Fellowship during the summer of 1995, when this work was completed, and all the members of that Institute for the warm hospitality and the profitable discussions with many of them. (P.G.G.) thanks Prof. F. Melchiorri of the University of Rome for having suggested the present study. We also thank Dr. Paidarova for sending us her computed dipole function for the $\mathrm{LiH}^{+}$molecule.

\section{References}

Bellini M., De Natale P., Inguscio M., Fink E., Galli D., Palla F., 1994, ApJ 424, 507 
Ben-Shlomo S., Kaldor U., 1988, J. Chem. Phys. 89, 956

Berriche H., 1995, Thèse d'État, Université Paul Sabatier, Toulouse, France

Berriche H., Gadea F.X., 1995, Chem. Phys. 191, 119

Boutalib A., Gadea F.X., 1992, J. Chem. Phys. 97, 1144

Chan Y.C., Harding D.R., Stwalley W.C., Vidal C.R., 1986, J. Chem. Phys. 85, 2436

Dalgarno A., Kirby K., Stancil P.C., 1995, ApJ (accepted)

Dalgarno A., Lepp S., 1987, Astrochemistry, IAU symposium 118. In: Tarafdar S.P. and Varshni M.P. (eds.). Dordrecht, Reidel, p. 109

De Bernardis P., Dubrovich V., Encrenaz P., et al., 1993, A\&A 269, 1

Docken K.K., Hinze J., 1972, J. Chem. Phys. 57, 4928 (D \& $\mathrm{H})$

Dyer S., Steiner E., 1986, Mol. Phys. 59, 1027

Foucrault M., Millie P., Daudey J.P., 1992, J. Chem. Phys. 96, 1257

Gianturco F.A., Gallese F., 1988, Chem. Phys. Lett. 148, 365

Gianturco F.A., Patriarca M., Roncero O., 1989, Mol. Phys. 67,281

Grabandt O., Bakker H.J., de Langes C. A., 1992, Chem. Phys. Lett. 189, 291

Huber K.P., Herzberg G., 1978, Mol. Spect. Mol. Struct. 4, Van Nostrand, Princeton

Hutchins J.B., 1976, ApJ 205, 103

Klopper W., Kurtzelnigg W., 1991, J. Chem. Phys. 94, 22

Lepp S., Shull J.M., 1984, ApJ 280, 465

Maoli R., Melchiorri F., Tosti D., 1994, ApJ 425, 372
Mendez L., Cooper I.L., Dickinson A.S., Mo O., Riera A., 1990, J. Phys. B. 23, 2797

Mo Y., Wu W., Zhang Q., 1993, J. Mol. Struct. 283, 237

Morgan T.J., Olson R.E., Schlachter A.S., Gallagher J.W., 1985, J. Phys. Chem. Ref. Data 14, 971

Ogilvie J.F., Oddershade J., Sauer S.P.A., 1994, Chem. Phys. Lett. 228, 183

Palke W.E., Goddard W.A., 1969, J. Chem. Phys. 50, 4524

Partridge H., Langhoff S.R., 1981, J. Chem. Phys. 74, 2361 $(\mathrm{P} \& \mathrm{~L})$

Peebles P.J.E., 1971, Physical Cosmology. Princeton University Press, Princeton

Rothstein E., 1969, J. Chem. Phys. 50, 1899

Sasagane K., Mori K., Ichiharaond A., Itoh R., 1990, J. Chem. Phys. 92, 3619

Stancil P.C., Lepp S., Dalgarno A., 1995, ApJ (accepted)

Stwalley W.C., Zemke W.T., 1993, J. Phys. Chem. Ref. Data 22,87

Tortorelli J.J., Harriman J.E., 1978, J. Chem. Phys. 69, 3163

Voitik J., Cespiva L., Savrda J., Paidarova I., 1990, J. Mol. Spectr. 142, 279

Wagoner R.V., Fowler W.A., Hoyle F., 1967, ApJ 148, 3

Wang X.C., Freed K.F., 1989, J. Chem. Phys. 91, 3002

Way K.R., Stwalley W.C., 1973, J. Chem. Phys. 59, 5298

Wharton L., Gold L.P., Klemperer W., 1960, J. Chem. Phys. 33,1255

Wiese W.L., Smith M.V., Glennon B.M., 1966, Atom. Trans. Prob. 1, Nat. Bureau of Standards, Washington D.C.

Zemke W.T., Stwalley W.C., 1980, J. Chem. Phys. 73, 5584 